\title{
Bone morphogenetic protein 2 improves patellar tendon healing by promoting migration and proliferation of tenocytes
}

\author{
LI Zhen ${ }^{1,2,3}$, SHEN XiaoTao ${ }^{1,2,3}$, CAO Liang ${ }^{1,2,3}$, YUAN ZiQiang ${ }^{1,3,4}$, CHEN SiYun ${ }^{1,2,3}$, \\ ZHENG Xin $^{1,2,3}$, TANG MeiKuen ${ }^{1,2,5}$, LEE Kenneth K ${ }^{1,2,3,5}$ \& CAI DongQing ${ }^{1,2,3^{*}}$ \\ ${ }^{1}$ Key Laboratory for Regenerative Medicine of Ministry of Education, Jinan University, Guangzhou 510632, China; \\ ${ }^{2}$ Joint Laboratory for Regenerative Medicine, the Chinese University of Hong Kong-Jinan University, Guangzhou 510632, China; \\ ${ }^{3}$ International Base of Collaboration for Science and Technology (JNU), the Ministry of Science and Technology \& Guangdong Province, \\ Guangzhou 510632, China; \\ ${ }^{4}$ Department of Molecular Genetics, Albert Einstein College of Medicine of Yeshive University, New York 10461, USA; \\ ${ }^{5}$ School of Biomedical Sciences, the Chinese University of Hong Kong, Hong Kong SAR, China
}

Received November 24, 2010; accepted February 17, 2011

\begin{abstract}
The repair of injured tendons remains a great challenge because of the poor intrinsic healing capacity of tendons. In this study, we examined the spatiotemporal expression pattern of the bone morphogenetic protein $2(b m p-2)$ gene in normal and experimentally injured rat patellar tendons. We also investigated the ability of exogenously applied BMP-2 to promote patellar tendon healing. In situ hybridization with $b m p-2$ and alk-6 (bmp-2 receptor) antisense riboprobes revealed that both genes were normally expressed at low levels in intact rat tendons. However, $b m p-2$ expression was significantly upregulated in tenocytes found in the wound site at $7 \mathrm{~d}$ and later following tendon injury. In addition, it was found that $b m p-2$ was expressed in cultured patellar tenocytes. Application of exogenous BMP-2 to the tendon wound site significantly enhanced tendon repair. Moreover, in vitro and in vivo studies further demonstrated that BMP-2 enhanced tenocyte proliferation and migration to the wound site. The detectable amount of BMP-2 in normal tendons suggests that a basal level of $b m p-2$ expression was likely present to maintain the normal functions of the patellar tendon. Injury to the tendon induced increased $b m p-2$ expression intrinsically, but the expression level was insufficient for proper tendon repair. Our findings indicate that it is possible to significantly improve tendon healing by applying exogenous BMP-2 to the wound site, inferring that this protein could be developed as a potential therapeutic reagent for the treatment of damaged tendons.
\end{abstract}

BMP-2, tendon healing, tenocyte proliferation and migration

Citation: $\quad$ Li Z, Shen X T, Cao L, et al. Bone morphogenetic protein 2 improves patellar tendon healing by promoting migration and proliferation of tenocytes. Chinese Sci Bull, 2011, 56: 1361-1369, doi: 10.1007/s11434-011-4438-7

Torn ligaments and tendons are among the most commonly encountered injuries. The standard method of treatment is to use the patellar tendon as an autograft for reconstruction of the injured ligament [1-4]. However, clinical studies have revealed that the patellar tendon donor site heals very slowly following the extraction of a strip of tendon to create the autograft. Many other complications have also been identified, including patellofemoral pain, rupture of the

*Corresponding author (email: tdongbme@jnu.edu.cn) patellar tendon and weakness in the quadriceps skeletal muscles [5-7]. In animal studies, it has been reported that scar formation in the patellar tendon extract/wounded site was the main feature, and all new collagen fibers produced at the wounded site were rudimentary in size and randomly aligned. Furthermore, the mechanical load that these experimental tendons were able to sustain before tearing was significantly reduced when compared with control tendons [8]. Effective healing of the patellar tendon is most likely related to the following 2 key events that must occur at the 
cellular and molecular levels: (1) Tenocytes from intact regions of the injured patellar tendon must be able to proliferate and migrate into the wounded site; (2) once these cells have fully occupied the wounded site, they must be able to produce collagen fibers that structurally complement the rest of the fibers in the intact tendon. Therefore, we would like to establish a method that can enhance these key events for effective tendon healing. In the present study, we investigated the possibility of enhancing tendon repair by intervening in the natural healing process of the damaged tendon with exogenous bone morphogenetic protein 2 (BMP-2) in the rat model. We established the spatiotemporal expression pattern of bmp-2 and its receptor, alk-6, in normal and experimentally injured patellar tendons. We also determined the effects of exogenous BMP-2 on tenocyte proliferation and migration during the tendon healing process.

\section{Materials and method}

\subsection{Animal surgery}

Eight-week-old male Sprague-Dawley (SD) rats (Guangdong Medical Laboratory Animal Center, China) were anesthetized with $2.5 \%$ pentobarbital solution injected into the peritoneum. The hair on the lower limbs was shaved off at the level of the stifle, and the skin was sterilized with chlorhexidine (Hibitane). An incision was then made in the skin to expose the patellar tendon. A fragment of tendon measuring $1 \mathrm{~mm} \times 4 \mathrm{~mm}$ was surgically removed from the central region of the patellar tendon. The tendon sheath and skin were then sutured. As a sham control, the skin and tendon sheath of the contralateral limbs were exposed and then immediately sutured. The experimental and sham control patellar tendons were harvested 2, 7, 14, 40, and $60 \mathrm{~d}$ postoperatively for analysis. All of the surgical procedures performed were approved by the animal ethics committees of Jinan University and the Chinese University of Hong Kong.

\subsection{Histology}

All of the tendons recovered were fixed in $4 \%$ paraformaldehyde at $4^{\circ} \mathrm{C}$ overnight. The specimens were then dehydrated in a graded series of alcohol, cleared in xylene, and embedded in paraffin wax. The wax blocks were sectioned at $6 \mu \mathrm{m}$ and mounted onto TESPA-coated glass slides. The sections were used for routine histology and in situ hybridization studies. Hoechst 33342 (Sigma-Aldrich, USA) fluorescent stains were used to stain the nuclei to confirm the presence of cells in the wounded site.

\subsection{In situ hybridization}

Digoxigenin-labeled sense and antisense riboprobes were synthesized from a psP72 plasmid containing a 1550-bp insert encoding bmp-2 (kindly provided by B. Hogan) and a pcDNA3 plasmid containing 1200 bp encoding alk-6 (kindly provided by P. ten Dijike) using T3 and T7 RNA polymerases (Boehringer Mannheim Biochemica, USA). The size of the probes was reduced by hydrolysis in 40 mmol L $\mathrm{L}^{-1}$ sodium bicarbonate and $60 \mathrm{mmol} \mathrm{L}^{-1}$ sodium carbonate at $60^{\circ} \mathrm{C}$ for $15 \mathrm{~min}$. The probes were precipitated with $4 \mathrm{~mol} \mathrm{~L}^{-1} \mathrm{LiCl}$ and ethanol, then resuspended in hybridization buffer. For in situ hybridization, paraffin sections were dewaxed, hydrated, and equilibrated in PBS. The sections were then treated with $10 \mu \mathrm{g} / \mathrm{mL}$ proteinase $\mathrm{K}$ for $10 \mathrm{~min}$ and postfixed in 4\% paraformaldehyde. The treated specimens were washed twice with PBS for 10 min and then incubated in prehybridization buffer for $3 \mathrm{~h}$. Afterward, 1 $\mu \mathrm{g} / \mathrm{mL}$ digoxigenin-labeled sense or antisense riboprobes were diluted in hybridization buffer and added to the treated specimens. The sense probes were used as the control. The hybridization temperature for $b m p-2$ and alk- 6 sense and antisense riboprobes was $50^{\circ} \mathrm{C}$, and the incubation time was $18 \mathrm{~h}$. After hybridization, all unbound cDNA probes were stringently washed off in $2 \times \mathrm{SSC}$ at $42^{\circ} \mathrm{C}$ for $10 \mathrm{~min}(3$ changes), followed by $60 \%$ formamide in $0.2 \times \mathrm{SSC}$ buffer for $15 \mathrm{~min}$ and $0.2 \times \mathrm{SSC}$ buffer for $10 \mathrm{~min}$. The location of hybridized probes was determined using alkaline phosphatase-conjugated digoxigenin antibodies. The antibodies were added to the specimens for $1 \mathrm{~h}$, washed in PBS, and developed in NBT/BICP.

\subsection{Culture of tenocytes}

Patellar tendon explant cultures were prepared as previously described [9]. Briefly, 3-month-old male SD rats were sacrificed using a chloroform overdose. The patellar tendon was excised and immediately transferred to prewarmed Dulbecco's phosphate buffered saline (Sigma-Aldrich, USA) containing $0.4 \%$ bovine serum albumin. The excised tendon was divided into small pieces of approximately 1 $\mathrm{mm}^{3}$ each, and each piece was implanted into $5 \mu \mathrm{L}$ of $10 \%$ rat tail collagen gel containing Minimal Essential Medium (Sigma-Aldrich, USA). The explants were incubated at $37^{\circ} \mathrm{C}$ for $20 \mathrm{~min}$. Finally, the culture dishes were flooded with Dulbecco's Modification of Eagle's Medium (DMEM) (Gibco, USA) containing 10\% heat inactivated fetal bovine serum (FBS) (Gibco, USA). The cultures were maintained at $37^{\circ} \mathrm{C}$ in a humidified incubator containing a mixture of $5 \% \mathrm{CO}_{2}$ in air. The medium was changed every $3 \mathrm{~d}$ until a confluent monolayer was established. These primary cultures were subsequently passaged by incubation with $0.25 \%$ trypsin (Invitrogen, USA). Trypsin activity was inhibited by addition of $10 \%$ FBS in DMEM, and the cells were pelleted by centrifugation at $459 \times g$ for $5 \mathrm{~min}$ at room temperature. The pellet was resuspended in DMEM containing 10\% FBS and subcultured. The subcultured tenocytes were used in the RT-PCR, "scratch wound" assay, transwell motility assay, collagen matrix contract assay, and MTT assay. 


\subsection{RT-PCR}

For the RT-PCR, total RNAs were isolated from the isolated tenocytes using TRIzol Reagent (Invitrogen, USA). The first-strand cDNA was synthesized by using the First Strand cDNA Synthesis Kit (Fermentas, USA) according to the manufacturer's instructions. PCR was performed by using bmp-2 primers (Sense: 5'-AGCGGAAGCGTCTTAAGTCCAG-3'; antisense: 5'-AGGTGATCAGCCAGGGGAAAAG-3'), and the program was: denaturation at $94^{\circ} \mathrm{C}(5 \mathrm{~min})$, 35 cycles of denaturation at $95^{\circ} \mathrm{C}(15 \mathrm{~s})$, annealing at $55^{\circ} \mathrm{C}$ $(30 \mathrm{~s})$, and extension at $72^{\circ} \mathrm{C}(60 \mathrm{~s})$. The PCR products were visualized following electrophoresis on ethidiumbromide-stained $1.5 \%$ agarose gel.

\subsection{3-(4,5-dimethylthiazol-2-yl)-2,5-diphenyl (MTT) assay}

MTT assay was performed to measure the extent of tenocyte proliferation in the presence and absence of human recombinant BMP-2 $(0.6 \mu \mathrm{g} / \mathrm{mL})$ (R\&D Systems, USA) using the method described by Mosmann in 1983 [10]. Briefly, $50 \mu \mathrm{L}$ of MTT solution was added to each of the 96 wells containing tenocytes and incubated for $4 \mathrm{~h}$ at $37^{\circ} \mathrm{C}$. DMSO $(150 \mu \mathrm{L})$ (Sigma-Aldrich, USA) was then added into each of the wells for $10 \mathrm{~min}$ to solubilize the blue MTT-tetrazolium dye. The dye was measured on an ELISA plate reader (Molecular Device SpectraMax 340, USA) with the absorbance set at $595 \mathrm{~nm}$. The data were collected from 3 independent experiments.

\subsection{Cell cycle analysis by flow cytometry}

BMP-2 $(6 \mu \mathrm{g} / \mathrm{mL})$-treated and untreated patellar tenocytes were collected $48 \mathrm{~h}$ after culture. The cells were fixed in $4 \%$ paraformaldehyde and resuspended in $1 \mathrm{~mL}$ of PBS and $0.1 \%$ Triton X-100. Twenty microliters of RNAase (10 $\mathrm{mg} / \mathrm{mL}$ ) (Fermentas, USA) and $20 \mu \mathrm{L}$ of propidium iodide solution (MP biomedicals, USA) were then added to the cell suspension. The suspension was incubated at $37^{\circ} \mathrm{C}$ for 30 min in the dark. The stained cells were resuspended and passed through a $40-\mu \mathrm{m}$ mesh filter. A total of $10^{6}$ cells $/ \mathrm{mL}$ were used for flow cytometry analysis (BD-FACSAria, USA). An argon laser (488-nm wavelength) was used to excite the propidium-iodide-stained cells. The emitted fluorescent signals were collected in a photomultiplier tube with a red pass filter (620-nm wavelength). The results obtained were analyzed using ModFit LTTM Version 3.2 software (Verity Software House, USA) to determine the percentage of cells in the $G_{0} / G_{1}, S$, and $G_{2} / M$ phases of the cell cycle.

\subsection{Collagen matrix contraction assay}

The patellar tenocytes $\left(1 \times 10^{5}\right)$ were suspended in $2 \mathrm{~mL}$ of $2 \times$ DMEM containing $0.5 \mathrm{~mL} 100 \mathrm{mmol} \mathrm{L}^{-1} \mathrm{NaOH}$ and 0.5
$\mathrm{mL}$ gelatin-A sepharose-pretreated FCS. Collagen lattices were prepared in $30-\mathrm{mm}$ dishes mixing $2 \mathrm{~mL}$ of the tenocytes in $0.7 \mathrm{~mL}$ of rat tail tendon collagen solution (1 $\mathrm{mg} / \mathrm{mL}$ ) [11]. The tenocyte-populated collagen lattices (TPCLs) were allowed to set for $60 \mathrm{~min}$ at $37^{\circ} \mathrm{C}$. Two milliliters of $2 \%$ FCS/DMEM with BMP-2 $(0.6 \mu \mathrm{g} / \mathrm{mL})$ and without BMP-2 was added into individual TPCLs for further culture at $37^{\circ} \mathrm{C}$ and $5 \% \mathrm{CO}_{2}$. The extent to which the collagen lattice was contracted by the tenocytes was determined by measuring the diameter of the lattice. The average diameter of the lattice was determined 2,3 , and $7 \mathrm{~d}$ postculture and from 3 independent experiments.

\subsection{Scratch wound assay}

A scratch wound assay was used to determine the effects of BMP-2 on cell migration and proliferation at the "wounded" site in vitro. The patellar tenocytes were cultured in culture dishes with DMEM $+10 \%$ FBS until they were confluent and monolayered. A sterile pipette tip was then used to scrape the cells off the midline of the culture to create an artificial wound. The width of the "wound" $(5 \mathrm{~mm})$ was verified to be consistent under dissection in all experiments. The cultures were then washed in PBS and cultured in the presence of (1) DMEM + 2\% FBS or (2) DMEM+2\% FBS + BMP-2 $(0.6 \mu \mathrm{g} / \mathrm{mL})$. The cultures were photographed at various time points. The experiment was repeated independently 3 times.

\subsection{Transwell motility assay}

Migration assays were performed using Nucleopore Blindwell chemotaxis chambers (Costar Corporation, USA) and Nucleopore polycarbonate filters $(8-\mu \mathrm{m}$ pore size, Costar) [12]. The filters were precoated with $100 \mu \mathrm{g} / \mathrm{mL}$ human plasma fibronectin (Gibco, USA) overnight and then airdried. DMEM+BMP-2 $(0.6 \mu \mathrm{g} / \mathrm{mL})+2 \%$ FBS were introduced into the bottom well of each Blindwell chamber. A Nucleopore filter was placed over the well and securely held in a retainer, forming the upper chamber. A suspension of patellar tenocytes $\left(10^{5}\right.$ cells) suspended in DMEM $+2 \%$ FBS was added to the upper chamber. The cells were cultured in the Blindwell chamber at $37^{\circ} \mathrm{C}$ and $5 \% \mathrm{CO}_{2}$ for 16 h. After culturing, the tenocytes were wiped off the upper surface of the filter with a cotton swab. The cells that had migrated to the underside of the filter were fixed, stained with eosin, and counted under a microscope. Each experiment was repeated 3 times.

\subsection{Preparation of BMP-2/alginate bead}

BMP-2 was loaded into alginate beads as follows. A $1.2 \%$ alginate solution was prepared by dissolving sodium alginate (Sigma-Aldrich, USA) in $2 \times$ DMEM. The mixture was then exposed to UV light for $12 \mathrm{~h}$ with stirring. A suitable 
amount of BMP-2 was added into the sterilized alginate mixture to obtain a final concentration of $2 \mu \mathrm{g}$ BMP- $-2 / \mathrm{mL}$. Ten microliters of the BMP-2/alginate mixture was then introduced into $102 \mathrm{mmol} \mathrm{L}^{-1}$ sterilized $\mathrm{CaCl}_{2}$ to generate the BMP-2/alginate gel bead. All BMP-2/alginate beads were balanced in DMEM ( $\mathrm{pH}$ 7.4) for 30 min before transplantation in vivo.

\subsection{Statistical analysis}

All values are expressed as means \pm SD. The unpaired Student's $t$-test with the use of a 2-tailed distribution was used to calculate the statistical significance between the means of the 2 groups. A $P$-value of $<0.05$ was considered to be significant.

\section{Results}

\section{$2.1 \quad b m p-2$ expression profile during tendon healing}

The spatiotemporal expression patterns of $b m p-2$ and its receptor, alk-6, were established during the tendon healing process by in situ hybridization. It was found that bmp-2 was normally expressed at a relatively low level in the nonoperated patellar tendons (Figure 1(b)). There was no staining in sections hybridized with the sense control riboprobe (Figure 1(a)). In 2-d postinjured tendons, fibroblast-like cells were found to infiltrate the wounded site (Figure 1(c) and (g)). These cells and the tenocytes in the intact regions of the tendon also expressed the gene, but in low levels (Figure 1(c)). In contrast, when the injured tendons were examined after $7 \mathrm{~d}$, the fibroblast-like cells in the wounded site strongly expressed bmp-2. Furthermore, the intensity of the expression was greater than that in the tenocytes of the unoperated tendon (Figure 1(d)). Two weeks postinjury, the cells in the wounded site still strongly expressed bmp-2, but this expression was weaker than that seen at $7 \mathrm{~d}$ postinjury (Figure 1(e) and (f)). Correspondingly, alk6 was expressed in exactly the same manner as that of bmp-2 at all time points examined (Figure 1(h); day 2 and 7 results not shown).

\section{$2.2 \quad b m p-2$ expression in cultured tenocytes}

The patellar tenocytes isolated from patellar tendons were cultured, and RT-PCR was applied to analyze the expression of the $b m p-2$ gene. It was found that cultured tenocytes expressed bmp-2 moderately (Figure 1(i)).

\subsection{Effect of BMP-2 on tenocyte proliferation in vitro}

Because the in situ hybridization results demonstrated that bmp-2 expression was increased in the healing patellar tendons, we tested whether BMP-2 could influence the proliferation of tenocytes in vitro. We isolated patellar tenocytes from the patellar tendon and cultured them in the presence of BMP-2. An MTT assay was used to determine the extent of cellular proliferation. The results showed that BMP-2 significantly enhanced tenocyte proliferation in vitro when compared with the untreated control cultures $(P<0.05)$ (Figure 2(a)). Furthermore, our flow cytometry analysis demonstrated that the number of S-phase tenocytes was
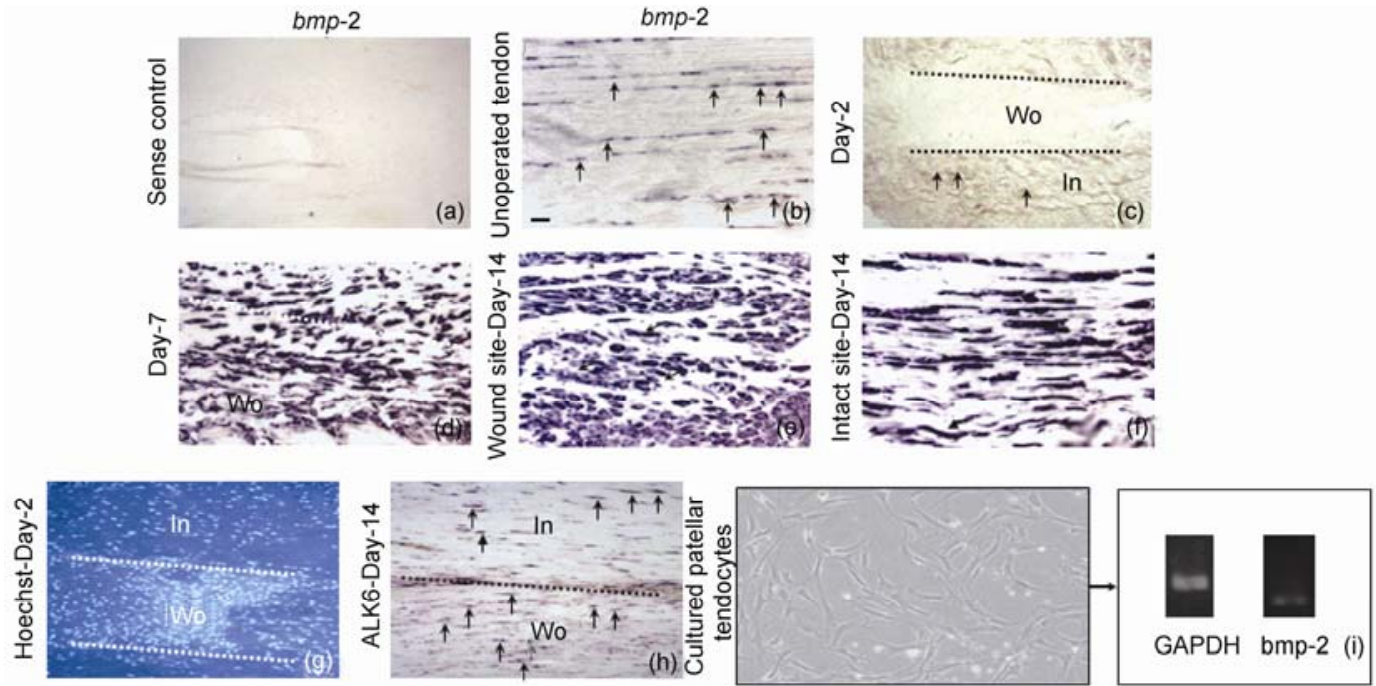

Figure 1 In situ hybridization showing bmp-2 (a-f) and alk-6 receptor (h) expression during tendon healing. (a) Sense control. (b) bmp-2 was normally expressed at low levels by tenocytes in unoperated patellar tendons (arrows). (c) In operated patellar tendons examined $2 \mathrm{~d}$ postoperatively, $b m p-2 \mathrm{was}$ found to be expressed by tenocytes (arrows) in the intact regions (In) and near the wounded site (Wo). In contrast, the cells that occupied the wounded site (Wo) did not express bmp-2. (d) In 7-d-old operated tendons, the tenocytes within the wounded site began to strongly express bmp-2 (arrows). Similarly, in 14-d-old operated specimens, the tenocytes continued to express bmp-2 in the wounded site (e) and the intact regions (f). (g) Nuclear Hoechst stain demonstrating the presence of cells within the wounded site of 2-day-old operated specimens. (h) alk-6 was coexpressed with bmp-2 in tenocytes within the wounded site and intact regions of 14-d-old operated specimens (arrows). The results were determined from 3 independent experiments. (i) $b m p-2$ was expressed in cultured patellar tenocytes. In, intact region; Wo, wounded site. 


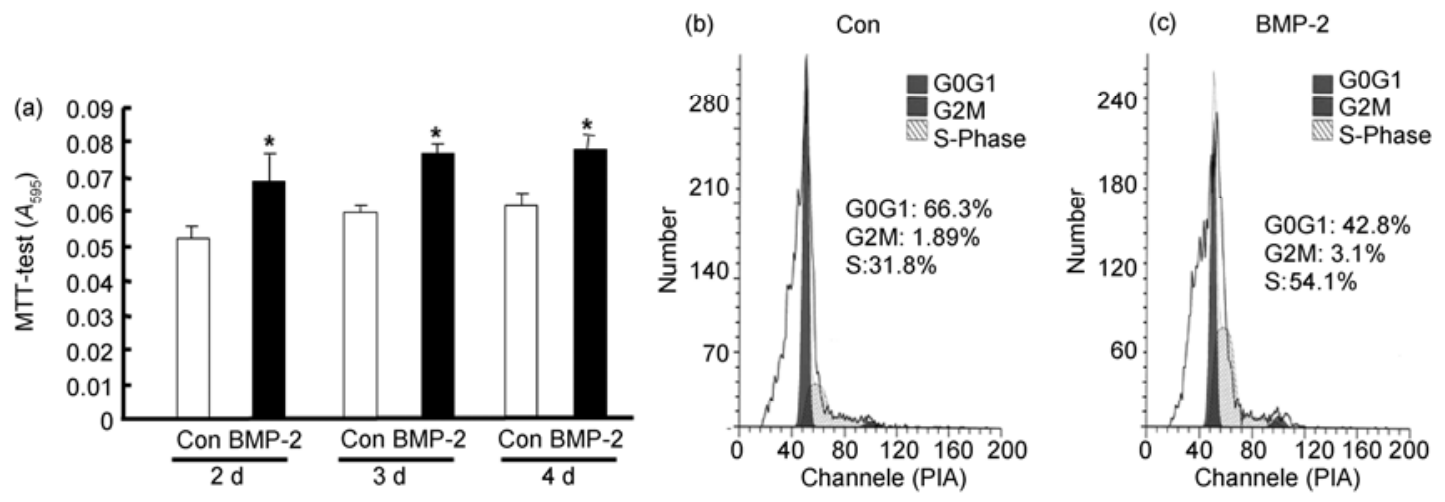

Figure 2 (a) MTT assay showing the effects of BMP-2 $(0.6 \mu \mathrm{g} / \mathrm{mL})$ on patellar tenocyte proliferation. *, $P<0.05$ (BMP-2 vs. Con). The results were determined from 3 independent experiments $(n=3)$. (b) Flow cytometry analysis revealed that the number of S phase in BMP-2 $(0.6 \mu \mathrm{g} / \mathrm{mL})$-treated patellar tenocytes was $54.1 \%$ after treatment with BMP-2 for $48 \mathrm{~h}$, while it was $31.8 \%$ in untreated controls. Con, control.

$54.1 \%$ after BMP-2 treatment for $48 \mathrm{~h}$, while it was $31.8 \%$ in the untreated controls (Figure 2(b)).

\subsection{Effects of BMP-2 on tenocyte-mediated contraction of collagen gel}

Collagen remodeling is one of the key steps in the tendon healing process. Hence, we tested whether BMP-2-treated patellar tenocytes inserted into a collagen lattice could enhance the contraction of the lattice- a representative phenomenon observed during extracellular matrix remodeling [13]. The patellar tenocytes were loaded into a drop of collagen gel, which was induced to solidify and allowed to free-float on the culture medium. The contractile kinetics of the tenocyte-collagen gels were recorded over a 7-d period. The results revealed that BMP-2 could not promote a significant increase in tenocyte- mediated contraction of collagen lattices $(P>0.05)$ (Figure 3$)$.

\subsection{Effects of BMP-2 on tenocyte migration in vitro}

Because cell migration is a critical step in the tendon healing process, we investigated whether BMP-2 is capable of influencing tenocyte movement. This was determined using a transwell assay and an artificial wound closure assay. In the transwell assay, BMP-2 was introduced into the bottom of the transwell chamber and tenocytes were placed in the upper chamber. A Millipore filter with pores that allowed cell migration was inserted between the 2 chambers. The results of the study showed that there were significantly more migrating tenocytes on the undersides of the Millipore filters in the BMP-2-treated samples than in the control samples $(P<$ 0.05 ) (Figure 4). We also used an artificial wound closure

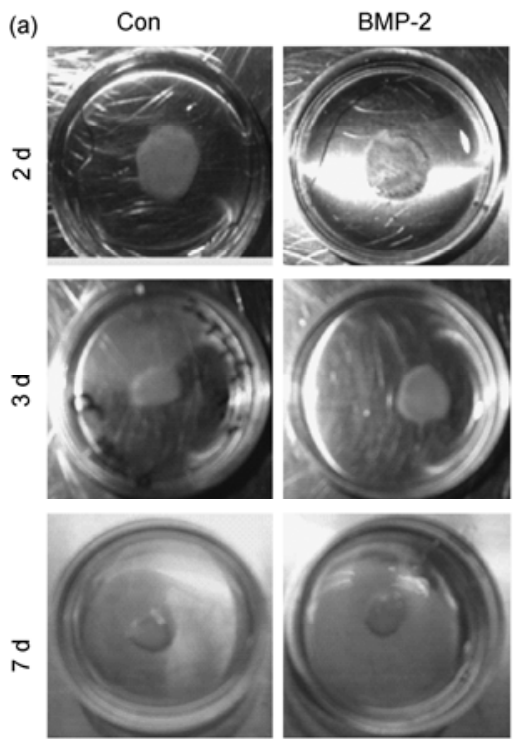

(b)

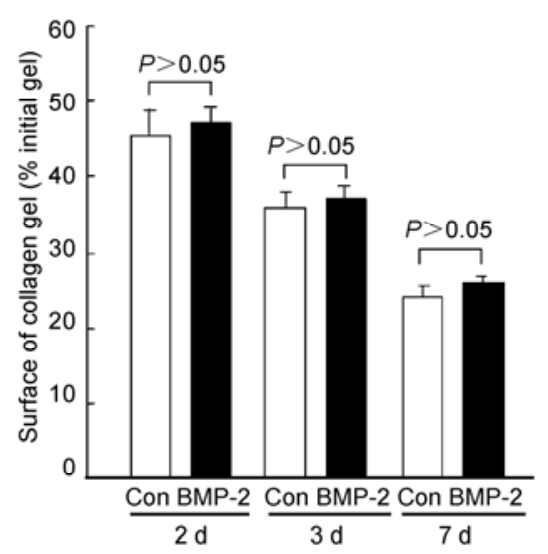

Figure 3 BMP-2 did not enhance the contraction of free-floating collagen lattices. Tenocytes were seeded into collagen lattices and allowed to float in the culture media (control) or medium containing BMP-2 $(0.6 \mu \mathrm{g} / \mathrm{mL}$ ) for 2 to $7 \mathrm{~d}$. (a) Photographs of a representative experiment; (b) graphic representation of the semi-quantification of the experiment shown in (a). Under such experimental conditions, the lattices contracted to the same extent between the BMP-2-treated group and the control group $(P>0.05)$. The results were established from 3 independent experiments $(n=3)$. 

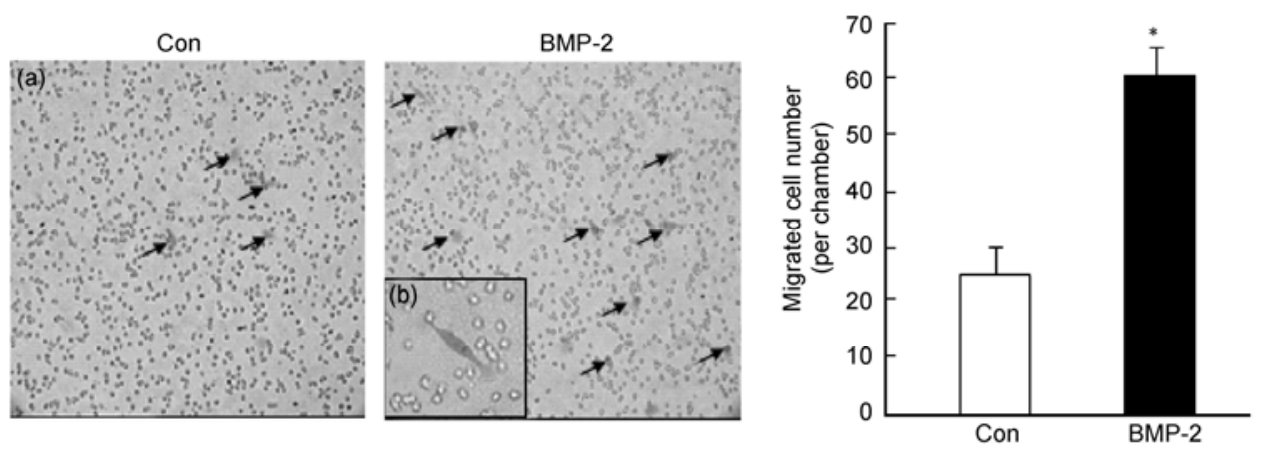

Figure 4 Transwell motility assay revealing that BMP-2 chemotactically attracted patellar tenocytes. The assay was performed in the absent (control) and presence of BMP-2 $(0.6 \mu \mathrm{g} / \mathrm{mL})$. (a) Representative results showing that there were significantly more tenocytes (arrows) present in the BMP-2 assay than in the control assay; (b) high-power image of the tenocytes that had migrated through the Millipore filters interposed between the 2 chambers making up the transwell apparatus; (c) bar chart of the semi-quantification of the experiments shown in (a). *, $P<0.05$ (BMP-2 vs. Con). Results established from 3 independent experiments $(n=3)$. Con, control.

assay, in which tenocytes were grown on culture dishes to confirm the findings. A 5-mm-wide scratch was created in the culture to mimic an artificial wound. The results of the experiment revealed that BMP-2-treated tenocytes migrated into the "wounded" site significantly faster than did untreated tenocytes. The BMP-2-treated tenocytes invaded approximately $70 \%$ of the "wound" within 72 h. In contrast, only approximately $40 \%$ of the "wound" site was filled with tenocytes in the untreated control cultures (Figure 5(a), (b), (e) and (f)).

\subsection{BMP-2 promotes tendon healing in vivo}

Our in vitro studies demonstrated that BMP-2 not only sig-
$\mathrm{Oh}$
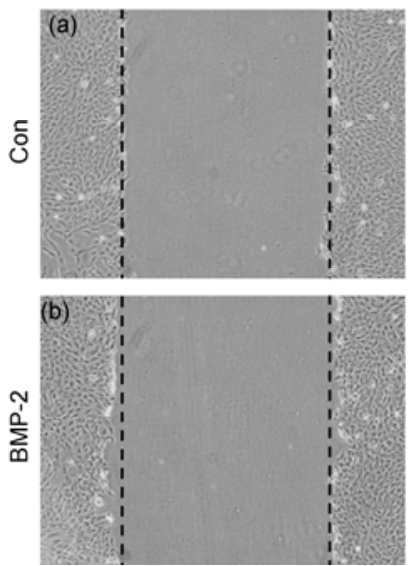

$72 \mathrm{~h}$
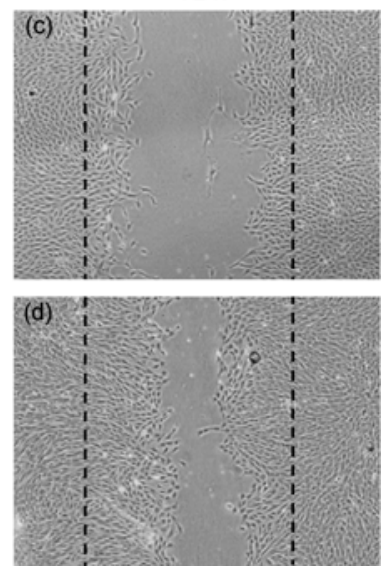

Figure 5 BMP-2 enhanced patellar tenocyte migration in the scratch wound assay. A 5-mm gap was scratched in a confluent tenocyte culture as a model to mimic a wound in an injured tendon. The culture was then maintained in DMEM + $2 \%$ FBS or DMEM + 2\% FBS + BMP-2. The gap/wound closure was monitored by microscopy. A representative micrograph for each treatment is shown, immediately $(0 \mathrm{~h})$ or $72 \mathrm{~h}$ after wounding. In the BMP-2-treated group, the tenocytes migrated into the gap/wound area and filled approximately $70 \%$ of the gap within $72 \mathrm{~h}$ (d). In contrast, only approximately $40 \%$ of the wound area was filled with tenocytes in control specimens (c). All of the experiments were repeated 3 times $(n=3)$. Con, Control. nificantly enhanced patellar tenocyte proliferation, but also migration. In addition, the in situ hybridization revealed that during the tendon healing process, bmp- 2 and alk- 6 expressions were upregulated in the cells that infiltrated the wounded site of the tendon. These results infer that BMP-2 may be indispensable during tendon repair and warranted further investigation in vivo, in a tendon injury animal model. We surgically created a wound in rat patellas and implanted alginate gel loaded with BMP-2 into the wounded site. As a control, only the medium used for suspending the BMP-2 was loaded into the alginate gel. The results of the study showed that the size of the wound in BMP-2-treated tendons was significantly smaller than that in the control tendons when examined 40 to $60 \mathrm{~d}$ after experimentation (Figure 6(a), (c)-(e)).

Histological examination also revealed that the wounds in the BMP-2-treated patellar tendons appeared to be almost repaired $40 \mathrm{~d}$ after injury. In contrast, a wide, open gap was still evident in the wounded site of the control tendon (Figure 6(e)). Histological examination of the BMP-2-treated tendons revealed that tenocytes migrated into the central area of the wounded site within $7 \mathrm{~d}$ postinjury, while significantly fewer tenocytes were found in the same region in the control tendon (Figure 6(b)). It is well known that BMP-2 can induce cells to differentiate into osteoblasts. However, under our experimental conditions, we found no signs of osteogenesis in the BMP-2-treated tendons when examined $60 \mathrm{~d}$ postinjury. This is consistent with our in vitro observations, which showed that BMP-2 promoted tenocyte migration and proliferation while showing no indications of osteogenesis.

\section{Discussion}

The patellar tendon is frequently used as an autograft in the reconstruction of the anterior cruciate ligament [3,4]. However, the drawback of this procedure is that the wounded site created in the donor's patellar tendon heals very slowly 

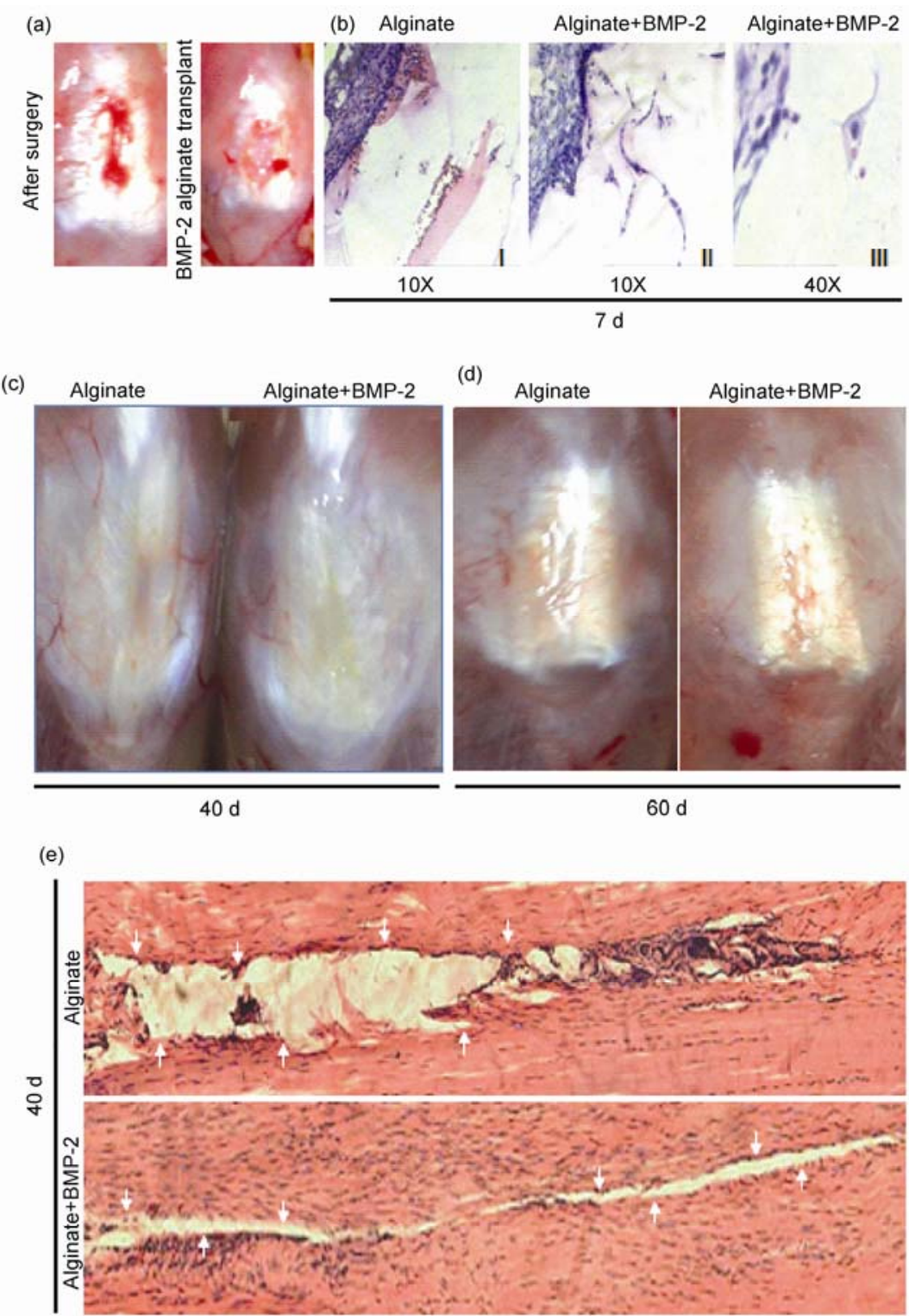

Figure 6 BMP-2 promoted patellar tendon healing in vivo. (a) Wound created in the patellar tendon and implanted with alginate gel loaded with BMP-2 protein. (b) H\&E staining of the experimental tendon $7 \mathrm{~d}$ after implantation of the alginate gel (I; 10×) and alginate gel loaded with BMP-2 (II for 10×; III for 40x). (c) and (d) The appearance of experimental tendons 40 to $60 \mathrm{~d}$ after implantation of alginate gel and alginate gel loaded with BMP-2, respectively. (e) Histology showing the size of the wound in a BMP-2-treated experimental tendon was significantly smaller compared with the alginate control (a, c, $d$ and e). Tenocytes were evident within the wounded site of BMP-2-treated tendons within $7 \mathrm{~d}$ after injury, while fewer cells were present in the wounds of control tendons (b). In addition, in the BMP-2-treated tendons, the wounds appeared almost completely healed within $40 \mathrm{~d}$ after injury (arrows), while a large gap (arrows) was still evident in the control tendons (e). All of the experiments were repeated 3 times $(n=3)$.

after surgery, resulting in many clinical complications. Therefore, it is important to identify a new therapeutic method that can help promote the healing of the injured tendon and minimize clinical complications.

BMPs belong to a family of multifunctional secreted proteins. Structurally, they are related to transforming growth factor- $\beta$ and activin [14-16]. BMPs were originally identified on the basis of their remarkable ability to induce bone and cartilage formation when implanted into the skeletal muscles of adult rats $[16,17]$. To date, 15 BMPs have been characterized and cloned [18]. Recent studies have demonstrated that physiologically, BMP-2 plays a pivotal role in many developmental processes, including osteoclast formation [19], chondrogenesis [20,21], myogenesis [22,23], skeletal development [24,25], apoptosis [26] and cardiogenesis [27-29]. bmp-2 null mutants have defective amnions, chorions, and hearts, and are nonviable [27]. In addition, ectopic expression of bmp-2 could promote fractured bone healing and tendon-to-bone healing [30,31]. Recently, it was reported that BMP-2 was involved in modu- 
lating the fate of tendon stem/progenitor cells [32,33].

In the present study, we examined the expression patterns of the bmp-2 gene in normal intact and experimentally damaged patellar tendons. Our results showed clear evidence that bmp-2 is normally expressed at low levels by tenocytes in intact tendons. In addition, we further documented that $b m p-2$ was expressed in cultured patellar tenocytes. The detectable amount of BMP-2 in normal tendons suggests that a basal level of bmp-2 expression was likely present to maintain the normal functions of the patellar tendon. These findings led us to investigate whether BMP-2 plays a role in the proliferation of tenocytes. Indeed, the MTT and flow cytometry results revealed that BMP-2 was able to increase the proliferation of tenocytes.

Furthermore, our in situ study also showed that following injury, bmp-2 expression was strongly upregulated among patellar tenocytes along the borders of the wounded site. It is possible that the expression of BMP-2 and its receptor may increase in the whole tendon after injury rather than just at the wounded site; these alterations could be a compensatory response or a response to inducing factors, which could migrate or present throughout all tissues. Alternatively, it could be a bystander effect, which may affect cells distal from the injured sites. It appears that in cultured tenocytes, the expression of BMP-2 was similar to that of the in vivo tenocytes. Previously, we reported that the cells that invaded the wounded area were tenocytes that had mainly originated from the edges of the wound [34]. Therefore, we reasoned that BMP-2 may also act in a chemotactic fashion to stimulate the cells bordering the injury to migrate into the wounded site. In this study, we demonstrated that BMP-2 not only significantly stimulated the tenocytes to migrate in the transwell model, but also significantly promoted the tenocytes to migrate earlier and faster into the artificial wounds that had been created in our "scratch wound" culture assay. In addition, we revealed that BMP-2 could promote the migration of tenocytes into injury sites in vivo in an animal model. These in vitro and in vivo results clearly demonstrated that BMP-2 was able to stimulate the migration of tenocytes.

Interestingly, it was found that tenocytes in the intact sites were expressing bmp-2, and once the tenocytes were stimulated to migrate into the injury site in vivo, they did not express bmp-2 as demonstrated in our in situ study. However, $7 \mathrm{~d}$ after injury, these cells strongly re-expressed bmp-2 as well as its receptor, alk-6. These observations suggest that BMP-2 is an important factor that is required for proliferation and migration of tenocytes. In contrast, BMP-2 appears not to be involved in collagen remodeling; the results of the tenocyte-mediated contraction assay revealed that BMP-2 could not promote a significant increase in tenocyte-mediated contraction of collagen lattices.

To investigate whether exogenous BMP-2 could also promote tendon healing in vivo, we transplanted alginate gels loaded with BMP-2 into the wounded sites of experi- mentally injured tendons. It was demonstrated that the size of the wounds in BMP-2-treated tendons was significantly smaller than that in untreated control specimens when examined at $40 \mathrm{~d}$ postinjury. A majority of the BMP-2-treated tendons appeared almost morphologically normal in the 40-d post-injured specimens, while wide, gaping wounds were still evident in the control tendons. These findings further demonstrated that exogenous BMP-2 was able to promote tendon healing. The fact that BMP-2 could increase the proliferation and migration of tenocytes suggests that there are many beneficial effects in the use of BMP-2 to improve tendon healing.

In summary, our data demonstrated that BMP-2 could promote tenocyte migration and proliferation and enhance effective repair of experimentally injured tendons in vivo. One of the major concerns of therapeutic application is the fear that BMP-2 could induce osteogenesis when implanted into damaged tendons [35]. On the contrary, we demonstrated that this was not the case, and that BMP-2 in fact promoted tenogenesis. These findings indicate that BMP-2 is a potential candidate for improving the poor healing capacity of damaged tendons.

This work was supported by the National Natural Science Foundation of China (30770886, 30570369, 30340038, 30973158), National High Technology Research and Development Program of China (2007AA02Z105), Natural Science Foundation of Guangdong Province (04105826), Key Science and Technology Program of Guangdong Province (2004B30601007), Key Science and Technology Program of Guangzhou (2006Z3E5251) and the Fundamental Research Funds for the Central Universities (21609408).

1 Hui C, Salmon L J, Kok A, et al. Fifteen-year outcome of endoscopic anterior cruciate ligament reconstruction with patellar tendon autograft for "isolated" anterior cruciate ligament tear. Am J Sports Med, 2011, 39: 89-98

2 Barber F A, Aziz-Jacobo J, Oro F B. Anterior cruciate ligament reconstruction using patellar tendon allograft: An age-dependent outcome evaluation. Arthroscopy, 2010, 26: 488-493

3 Noyes F R, Barber-Westin S D. Anterior cruciate ligament reconstruction with autogenous patellar tendon graft in patients with articular cartilage damage. Am J Sports Med, 1997, 25: 626-634

4 Arnoczky S P, Warren R F, Ashlock M A. Replacement of the anterior cruciate ligament using a patellar tendon allograft: An experimental study. J Bone Joint Surg Am, 1986, 68: 376-385

5 Adriani E, Mariani P P, Maresca G, et al. Healing of the patellar tendon after harvesting of its mid-third for anterior cruciate ligament reconstruction and evolution of the unclosed donor site defect. Knee Surg Sports Traumatol Arthrosc, 1995, 3: 138-143

6 Marumoto J M, Mitsunaga M M, Richardson A B, et al. Late patellar tendon ruptures after removal of the central third for anterior cruciate ligament reconstruction: A report of two cases. Am J Sports Med, 1996, 24: 698-701

7 Schippinger G, Passler J M, Seibert F J, et al. Are complications in cruciate ligament replacement operations with patellar tendon transplantation dependent on surgical technique and surgical timing? Swiss Surg, 1997, 3: 154-159

8 Kamps B S, Linder L H, DeCamp C E, et al. The influence of immobilization versus exercise on scar formation in the rabbit patellar tendon after excision of the central third. Am J Sports Med, 1994, 22: 803-811 
9 Chan B P, Chan K M, Maffulli N, et al. Effect of basic fibroblast growth factor: An in vitro study of tendon healing. Clin Orthop Relat Res, 1997, 342: 239-247

10 Mosmann T. Rapid colorimetric assay for cellular growth and survival: Application to proliferation and cytotoxicity assays. J Immunol Methods, 1983, 65: 55-63

11 Cook H, Davies K J, Harding K G, et al. Defective extracellular matrix reorganization by chronic wound fibroblasts is associated with alterations in TIMP-1, TIMP-2 and MMP-2 activity. J Invest Dermatol, 2000, 115: 225-233

12 Webb S E, Lee K K, Tang M K, et al. Fibroblast growth factors 2 and 4 stimulate migration of mouse embryonic limb myogenic cells. Dev Dyn, 1997, 209: 206-216

13 Celeste A J, Iannazzi J A, Taylor R C, et al. Identification of transforming growth factor beta family members present in bone-inductive protein purified from bovine bone. Proc Natl Acad Sci USA, 1990, 87: 9843-9847

14 Tachi K, Takami M, Sato H, et al. Enhancement of bone morphogenetic protein-2-induced ectopic bone formation by transforming growth factor- $\beta 1$. Tissue Eng Part A, 2011, 17: 597-606

15 Kingsley D M. The TGF-beta superfamily: New members, new receptors, and new genetic tests of function in different organisms. Genes Dev, 1994, 8: 133-146

16 Wozney J M, Rosen V, Byrne M, et al. Growth factors influencing bone development. J Cell Sci Suppl, 1990, 13: 149-156

17 Blader P, Rastegar S, Fischer N, et al. Cleavage of the BMP-4 antagonist chordin by zebrafish tolloid. Science, 1997, 278: 1937-1940

18 Croteau S, Rauch F, Silvestri A, et al. Bone morphogenetic proteins in orthopedics: From basic science to clinical practice. Orthopedics, 1999, 22: 686-695, quiz 696-697

19 Chen D, Harris M A, Rossini G, et al. Bone morphogenetic protein 2 (BMP-2) enhances BMP-3, BMP-4, and bone cell differentiation marker gene expression during the induction of mineralized bone matrix formation in cultures of fetal rat calvarial osteoblasts. Calcif Tissue Int, 1997, 60: 283-290

20 De Luca F, Barnes K M, Uyeda J A, et al. Regulation of growth plate chondrogenesis by bone morphogenetic protein-2. Endocrinology, 2001, 142: 430-436

21 Duprez D, Bell E J, Richardson M K, et al. Overexpression of BMP-2 and BMP-4 alters the size and shape of developing skeletal elements in the chick limb. Mech Dev, 1996, 57: 145-157

22 Katagiri T, Yamaguchi A, Komaki M, et al. Bone morphogenetic protein-2 converts the differentiation pathway of $\mathrm{C} 2 \mathrm{C} 12$ myoblasts into the osteoblast lineage. J Cell Biol, 1994, 127: 1755-1766

23 Katagiri T, Akiyama S, Namiki M, et al. Bone morphogenetic protein-2 inhibits terminal differentiation of myogenic cells by suppressing the transcriptional activity of MyoD and myogenin. Exp Cell Res, 1997, 230: 342-351

24 Kingsley D M, Bland A E, Grubber J M, et al. The mouse short ear skeletal morphogenesis locus is associated with defects in a bone morphogenetic member of the TGF beta superfamily. Cell, 1992, 71: 399-410

25 Kingsley D M. What do BMPs do in mammals? Clues from the mouse short-ear mutation. Trends Genet, 1994, 10: 16-21

26 Hay E, Lemonnier J, Fromigue O, et al. Bone morphogenetic protein-2 promotes osteoblast apoptosis through a Smad-independent, protein kinase C-dependent signaling pathway. J Biol Chem, 2001, 276: 29028-29036

27 Zhang H, Bradley A. Mice deficient for BMP2 are nonviable and have defects in amnion/chorion and cardiac development. Development, 1996, 122: 2977-2986

28 Chang H, Huylebroeck D, Verschueren K, et al. Smad5 knockout mice die at mid-gestation due to multiple embryonic and extraembryonic defects. Development, 1999, 126: 1631-1642

29 Yang X, Castilla L H, Xu X, et al. Angiogenesis defects and mesenchymal apoptosis in mice lacking SMAD5. Development, 1999, 126: $1571-1580$

30 Wang E A, Rosen V, D'Alessandro J S, et al. Recombinant human bone morphogenetic protein induces bone formation. Proc Natl Acad Sci USA, 1990, 87: 2220-2224

31 Martinek V, Latterman C, Usas A, et al. Enhancement of tendon-bone integration of anterior cruciate ligament grafts with bone morphogenetic protein-2 gene transfer: A histological and biomechanical study. J Bone Joint Surg Am, 2002, 84-A: 1123-1131

32 Bi Y, Ehirchiou D, Kilts $\mathrm{T} \mathrm{M}$, et al. Identification of tendon stem/progenitor cells and the role of the extracellular matrix in their niche. Nat Med, 2007, 13: 1219-1227

33 Rui Y F, Lui P P, Ni M, et al. Mechanical loading increased BMP-2 expression which promoted osteogenic differentiation of tendonderived stem cells. J Orthop Res, 2011, 29: 390-396

34 Lee K K, Cai D Q, Tang M K, et al. Growth arrest-specific 2 gene expression during patellar tendon healing. Cells Tissues Organs, 2003, 173: $138-146$

35 Ryoo H M, Lee M H, Kim Y J. Critical molecular switches involved in BMP-2-induced osteogenic differentiation of mesenchymal cells. Gene, 2006, 366: 51-57

Open Access This article is distributed under the terms of the Creative Commons Attribution License which permits any use, distribution, and reproduction in any medium, provided the original author(s) and source are credited. 